\title{
Graph Repair and its Application to Meta-Modeling*
}

\author{
Christian Sandmann \\ Universität Oldenburg \\ Oldenburg, Germany \\ christian.sandmann@uni-oldenburg.de
}

\begin{abstract}
Model repair is an essential topic in model-driven engineering. We present typed graph-repair programs for specific conditions; application to any typed graph yields a typed graph satisfying the condition. A model graph based on the Eclipse Modeling Framework (EMF), short EMF-model graph, is a typed graph satisfying some structural EMF-constraints. Application of the results to the EMF-world yields model-repair programs for EMF $k$ constraints, a first-order variant of EMF constraints; application to any typed graph yields an EMFk model graph. From these results, we derive results for EMF model repair.
\end{abstract}

\section{Introduction}

In model-driven software engineering, the primary artifacts are models [23, 8]. Models have to be consistent w.r.t. a set of constraints, specified for example in the Object Constraint Language (OCL) [15]. To increase the productivity of software development, it is necessary to automatically detect and resolve inconsistencies arising during the development process (see, e.g. [14, 11, 13]).

To enable automated model repair or model completion, we look for an algorithm that - given a metamodel with two constraints and any model satisfying one of the constraints - creates another model satisfying the old as well as a new one (see Figure 1).

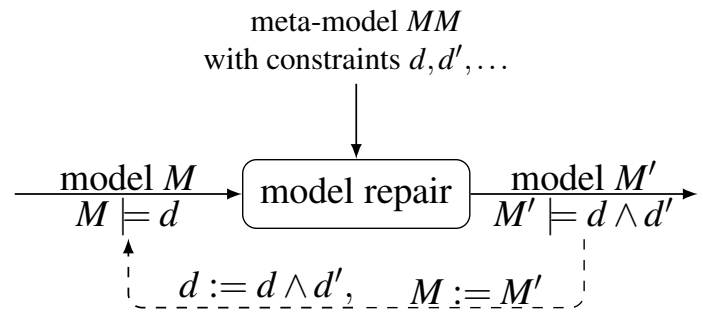

Figure 1: General idea to model repair

If we have such an algorithm, the process can be iterated: Using the model satisfying two constraints, and a new constraint as input, the algorithm creates a model satisfying the conjunction of three constraints, and so on. This iterative approach is necessary in handling large conditions. In each step, one condition is handled. If all steps terminate, and in all steps the preceding conditions remain preserved, we can

*This work is partly supported by the German Research Foundation (DFG), Grants HA 2936/4-2 and TA 2941/3-2 (MetaModeling and Graph Grammars: Generating Development Environments for Modeling Languages).

B. Hoffmann and M. Minas (Eds.): Eleventh International Workshop on Graph Computation Models (GCM 2020)

EPTCS 330, 2020, pp. 13-34 doi 10.4204/EPTCS.330.2
(C) Christian Sandmann

This work is licensed under the Creative Commons Attribution License. 
be sure that, after the consideration of all finitely many conditions, the conjunction of the conditions is satisfied. If after one step a preceding condition is violated, the condition must be considered again and the process may be come non-terminating.

In this paper, we represent a meta-model as a type graph, the instance model as a graph typed over the type graph, and first-order constraints as a typed graph constraint, equivalent to a first-order graph formula [20]. Given a typed constraint, we extract a typed program from the constraint, called repair program, such that the application of the typed repair program to an arbitrary typed graph yields a typed graph satisfying the constraint.

For small (basic) constraints, we extract a basic repair program directly from the constraint. For larger (proper) constraints, the repair program is composed from basic repair programs. For generalized proper constraints repair programs for subconditions may be used for the construction. For conjunctive constraints we take repair programs for the components and compose them to a typed repair program, provided that there is a sequentialization that preserves the preceding constraints. For disjunctive constraints we need a repair program for one component. Altogether, for so-called legit constraints, we can construct a repair program. These constructions are done in the $\mathscr{M}$-adhesive category of typed graphs with $\mathscr{E}^{\prime}-\mathscr{M}$ pair factorization.

A model graph based on the Eclipse Modeling Framework (EMF) [24], short EMF model graph, is a typed graph satisfying some structural EMF constraints. Application of the results for typed graphs to the EMF world yields model completion programs for EMF $k$ constraints, a first-order version of the EMF constraints, such that the application to a typed graph yields an EMFk model graph. The results known from typed graph repair are applied to EMF $k$ model repair and EMF model repair.

The structure of the paper is as follows. In Section 2, we review the definitions of typed graphs, typed graph conditions, and typed graph programs. In Section 3 , we introduce the concept of typed repair programs and show that there are repair programs for a large number of conditions, so-called legit conditions. Application of a typed repair program to any typed graph yields a typed graph satisfying the constraint. In Section 4, application of the results to EMF-world yields model-repair and completion programs for EMF $k$ constraints, a first-order variant of EMF constraints. From these results, we derive results of EMFmodel repair and completion. In Section 5, we present some related concepts. In Section 6, we give a conclusion and mention some further work.

\section{Preliminaries}

In the following, we recall the definitions of typed graphs, graph conditions, rules and transformations, graph programs, and basic transformations [2, 6, 17]. In the following, our concepts are based on [2]. For simplicity, we ignore the attributes.

A directed graph consists of a set of nodes and a set of edges where each edge is equipped with a source and a target node.

Definition 1 (graphs \& morphisms). A (directed) graph $G=\left(V_{G}, E_{G}, \mathrm{~s}_{G}, \mathrm{t}_{G}\right)$ consists of a set $V_{G}$ of nodes and a set $E_{G}$ of edges, as well as source and target functions $\mathrm{s}_{G}, \mathrm{t}_{G}: E_{G} \rightarrow V_{G}$. Given graphs $G$ and $H$, a (graph) morphism $g: G \rightarrow H$ consists of total functions $g_{V}: V_{G} \rightarrow V_{H}$ and $g_{E}: E_{G} \rightarrow E_{H}$ that preserve sources and targets, that is, $g_{V} \circ \mathrm{s}_{G}=\mathrm{s}_{H} \circ g_{E}$ and $g_{V} \circ \mathrm{t}_{G}=\mathrm{t}_{H} \circ g_{E}$. The morphism $g$ is injective (surjective) if $g_{\mathrm{V}}$ and $g_{\mathrm{E}}$ are injective (surjective), and an isomorphism if it is injective and surjective. In the latter case, $G$ and $H$ are isomorphic, denoted by $G \cong H$. 
Convention. Drawing a graph, nodes are drawn as circles and edges as arrows. Arbitrary morphisms are drawn by usual arrows $\rightarrow$, injective ones by $\hookrightarrow$.

A type graph (with containment) is a graph with a distinguished set of containment edges, and a relation of opposite edges.

Definition 2 (Type graph). A type graph $T G=(T, C, O)$ consists of a graph $T$, a set $C \subseteq E_{T}$ of containment edges, and a relation $O \subseteq E_{T} \times E_{T}$ of opposite edges. The relation $O$ is anti-reflexive, symmetric, functional, i.e., $\forall\left(e_{1}, e_{2}\right),\left(e_{1}, e_{3}\right) \in O, e_{2}=e_{3}$, and opposite direction, i.e., $\forall\left(e_{1}, e_{2}\right) \in O, \mathrm{~s}\left(e_{1}\right)=\mathrm{t}\left(e_{2}\right)$ and $\mathrm{s}\left(e_{2}\right)=\mathrm{t}\left(e_{1}\right)$.

Convention. The drawing of a type graph is obtained from the underlying graph by marking every containment edge $\left(\longrightarrow\right.$ ) with a black diamond at the source, and adding, for every pair $\left(e_{1}, e_{2}\right)$ of opposite edges, a bidirectional edge ( $\longleftrightarrow$ ) between the source and the target of the first edge with two edge type names, one at each end.

Example 1. A type graph for Petri-nets is given in Figure 2.

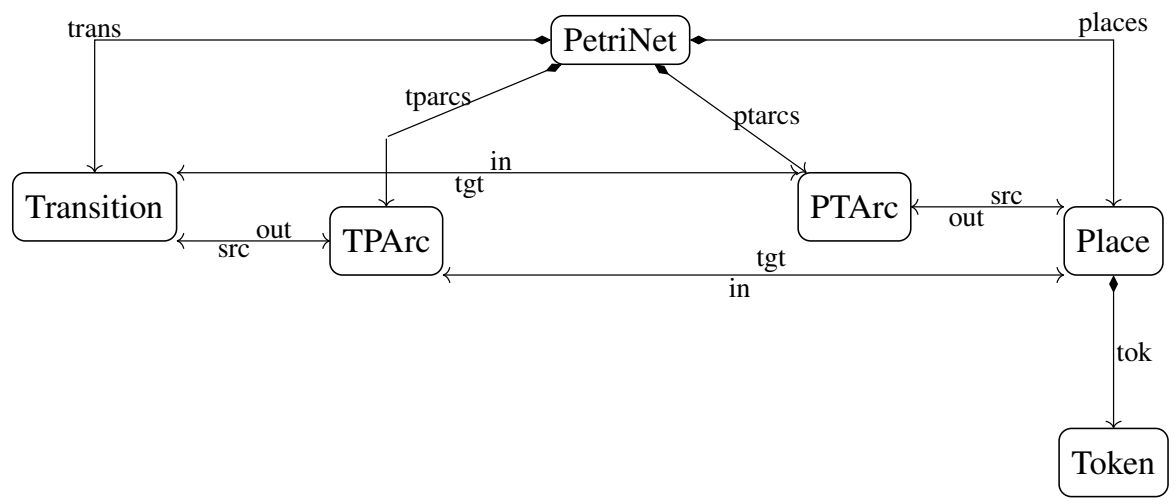

Figure 2: Type graph for Petri-nets, adapted from [27]

The type graph consists of the nodes PetriNet (PN), Place (Pl), Transition (Tr), Token (Tk), place-totransition arcs (PTArc), and transition-to-place arcs (TPArc), written inside the nodes, and the edges places, trans, and tok. The distinguished containment edges from the PetriNet to the Place (Transition, PTArc, and TPArc)-node are marked in the graph. The opposite edge relation relates the edges from the PTArc (TPArc)-node to the Place (Transition)-node of type src and the Place (Transition)-node to the PTArc (TPArc)-node of type out.

Assumption. In the following, let $T G=(T, C, O)$ be a fixed type graph.

A typed graph over a type graph is a graph together with a typing morphism. The typing itself is done by a graph morphism between the graph and the type graph.

Definition 3 (typed graphs). A typed graph (G,type) is a graph $G$ together with a typing morphism type: $G \rightarrow T$. Given typed graphs $\left(G\right.$, type $\left._{G}\right),\left(H\right.$, type $\left._{H}\right)$, a typed graph morphism $g: G \rightarrow H$ is a graph morphism such that $t_{y p e_{H, V}} \circ g_{V}=$ type $_{G, V}$ and type $e_{H, E} \circ g_{E}=$ type $_{G, E}$. For a node $v$ (an edge $e$ ) in $G$, type $_{V}(v)\left(\right.$ type $\left._{E}(e)\right)$ is the node (edge) type. 
Convention. Given a typed graph $(G$, type $)$, we draw the graph $G$ and put in type information: For a node $v$ in $G$, we depict the node type $\operatorname{type}_{V}(v)$ inside the node; for an edge $e$ in $G$, we depict the edge type $\operatorname{type}_{E}(e)$ near the target node of the edge $e$. Each edge with edge type containment edge is marked as a containment edge. For every pair of nodes whose type nodes are connected by an opposite edge, an opposite edge is added. For each pair $\left(v_{1}, v_{2}\right)$ of nodes in $G$ for which type $e_{V}\left(v_{1}\right)$, type $e_{V}\left(v_{2}\right)$ are connected by an opposite edge, a bidirectional edge between the nodes with two edge type names, one at each end, is added.

Assumption. In the following, all graphs are typed over $T G$ and all morphisms are injective.

Note. Typed graphs (over $T G$ ) with containment and morphisms form a category Graphs $\mathbf{T G}$. This is $\mathscr{M}$-adhesive and has a $\mathscr{E}^{\prime}-\mathscr{M}$ pair factorization [5, 3] where $\mathscr{M}$ is the class of injective morphisms and $\mathscr{E}^{\prime}$ is the class of pairs of jointly surjective morphisms. $\mathscr{M}$-adhesiveness implies the existence of pushouts (used in Definition 5 and Lemma 11; $\mathscr{E}^{\prime}-\mathscr{M}$ pair factorization is used in the shift construction in Lemma 1 .

Typed graph conditions are nested constructs, which can be represented as trees of morphisms equipped with quantifiers and Boolean connectives. Graph conditions and first-order graph formulas are expressively equivalent.

Definition 4 (typed graph conditions). A (typed graph) condition over a graph $A$ is of the form (a) true or $\exists(a, c)$ where $a: A \hookrightarrow C$ is a real inclusion morphism, i.e., $A \subset C$, and $c$ is a condition over $C$. (b) For a condition $c$ over $A, \neg c$ is a condition over $A$. (c) For conditions $c_{i}(i \in I$ for some finite index set $I$ ) over $A, \wedge_{i \in I} c_{i}$ is a condition over $A$. Conditions over the empty graph $\emptyset$ are called constraints. In the context of rules, conditions are called application conditions. Any morphism $p: A \hookrightarrow G$ satisfies true. A morphism $p$ satisfies $\exists(a, c)$ with $a: A \hookrightarrow C$ if there exists an morphism $q: C \hookrightarrow G$ such that $q \circ a=p$ and $q$ satisfies $c$.

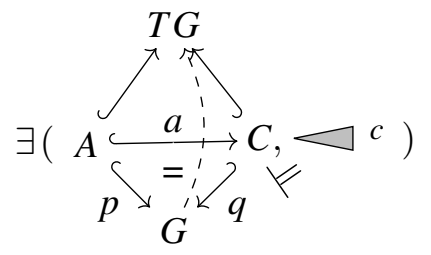

A morphism $p$ satisfies $\neg c$ if $p$ does not satisfy $c$, and $p$ satisfies $\wedge_{i \in I} c_{i}$ if $p$ satisfies each $c_{i}(i \in I)$. We write $p \models c$ if $p$ satisfies the condition $c$ (over $A$ ). A graph $G$ satisfies a constraint $c, G \models c$, if the morphism $p: \emptyset \hookrightarrow G$ satisfies $c$. A constraint $c$ is satisfiable if there is a graph $G$ that satisfies $c$.

Notation. Conditions may be written in a more compact form: $\exists a:=\exists(a$, true), false $:=\neg$ true, $\forall(a, c):=\nexists(a, \neg c)$, and $\nexists:=\neg \exists$. The expressions $\vee_{i \in I} c_{i}$ and $c \Rightarrow c^{\prime}$ are defined as usual. For a morphism $a: A \hookrightarrow C$ in a condition, we just depict the codomain $C$, if the domain $A$ can be unambiguously inferred.

The following is done in the framework of $\mathscr{M}$-adhesive categories. Rules are specified by a pair of morphisms, interface morphisms, and an application condition. By the interfaces, it becomes possible to hand over information between the transformation steps. In contrast to [25], our vertical morphisms are injective.

Definition 5 (typed rules \& transformations). Given a category $\mathscr{C}$, a (typed) rule $\rho=\langle x, p$, ac, $y\rangle$ (with interfaces $X, Y$ ) consists of a plain rule $p=\langle L \hookleftarrow K \hookrightarrow R\rangle$ of morphisms $l: K \hookrightarrow L, r: K \hookrightarrow R$, morphisms 
$x: X \hookrightarrow L, y: Y \hookrightarrow R$, the (left and right) interface morphisms, and a left application condition ac over $L$. The partial morphism $i: X \hookrightarrow Y$ with $i=y^{-1} \circ r \circ l^{-1} \circ x$ is the interface morphism of the rule $\rho$.

If the domain of an interface morphisms is empty or the application condition ac is true, then the component may not be written.

A direct transformation from $G$ to $H$ applying $\rho$ at $g: X \hookrightarrow G$ consists of the following steps:

(1) Mark a morphism $g^{\prime}: L \hookrightarrow G$, called match satisfying the dangling condition, such that $g=g^{\prime} \circ x$ and $g^{\prime} \models$ ac.

(2) Apply the plain rule $p$ at $g^{\prime}$ yielding a morphism $h^{\prime}: R \hookrightarrow H$.

(3) Unmark $h: Y \hookrightarrow H$, i.e., define $h=h^{\prime} \circ y$.

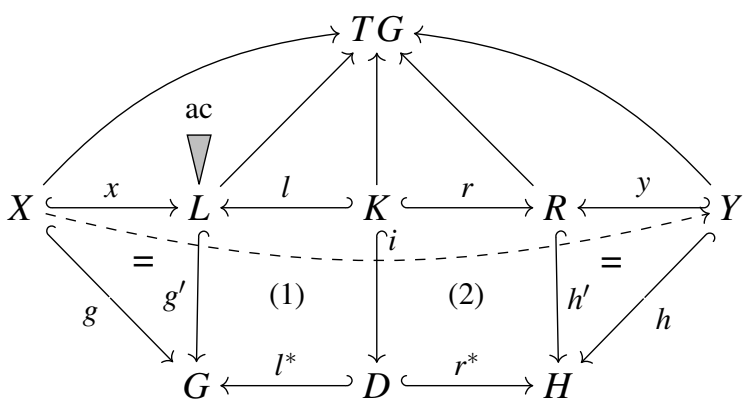

Figure 3: A direct transformation

The application of a plain rule is as in the double-pushout approach [3] in the category of typed graphs. A plain rule $p=\langle L \hookleftarrow K \hookrightarrow R\rangle$ is applicable to a graph $G$ w.r.t. a morphism $g^{\prime}: L \hookrightarrow G$, iff $g^{\prime}$ satisfies the dangling condition: "No edge in $G-g^{\prime}(L)$ is incident to a node in $g^{\prime}(L-K)$."

The semantics of the rule $\rho$ is the set $\llbracket \rho \rrbracket$ of all triples $\langle g, h, i\rangle$ of a morphism $g: X \hookrightarrow G$, a morphism $h: Y \hookrightarrow H$, and a partial interface morphism $i: X \hookrightarrow Y$ with $i=y^{-1} \circ r \circ l^{-1} \circ x$. Instead of $\langle g, h, i\rangle \in \llbracket \rho \rrbracket$, we write $g \Rightarrow_{\rho, i} h$ or short $g \Rightarrow_{\rho} h$.

The dangling edges operator. For node-deleting rules $\rho$, the dangling condition may not be satisfied. In this case, $\rho^{\prime}$ means that the rule shall be applied in the SPO-style of replacement [10], i.e., first to remove the dangling edges, and, afterwards application of the rule in the DPO style. Note that this style of replacement also can be described by a DPO-program that fixes a match for the rule, deletes the dangling edges, and afterwards applies the rule at the match. The proceeding can be extended to sets of rules: For a rule set $\mathscr{S}, \mathscr{S}^{\prime}=\left\{\rho^{\prime} \mid \rho \in \mathscr{S}\right\}$.

Interface \& markings. Rules with interfaces enable the control over marking and unmarking of elements in a typed graph and are capable of handling the markings over transformation steps. The left interface restricts the application of the rule to a previously marked context: Given a morphism $g: X \hookrightarrow G$, the application is restricted to those morphisms $g^{\prime}: L \hookrightarrow G$ that fit to $g$, i.e. $g=g^{\prime} \circ x$. The right interface restricts the application of the next rule: By the morphism $h: Y \hookrightarrow H$, the next rule can only be applied at $Y$. Instead of rules with interfaces in the sense of [17], we could use markings as, e.g., in [7, 18]. We have decided to use the interfaces instead of markings, because we have nested markings and the description of markings by morphisms makes transparent what happens. 
Typed graph programs are made of sets of typed rules with interface, non-deterministic choice $\{P, Q\}$, sequential composition $\langle P ; Q\rangle$, the try-statement try $P$, and the as long as possible iteration $P \downarrow$.

Definition 6 (typed graph programs). The set of (typed graph) programs with interface $X, \operatorname{Prog}(X)$, is defined inductively:

(1) Every typed rule $\rho$ with interface $X$ (and $Y$ ) is in $\operatorname{Prog}(X)$.

(2) If $P, Q \in \operatorname{Prog}(X)$, then $\{P, Q\}$ is in $\operatorname{Prog}(X)$.

(3) If $P \in \operatorname{Prog}(X)$ and $Q \in \operatorname{Prog}(Y)$, then $\langle P ; Q\rangle \in \operatorname{Prog}(X)$.

(4) If $P \in \operatorname{Prog}(X)$, then try $P$, and $P \downarrow$ are in $\operatorname{Prog}(X)$.

The statement Skip denotes the identity rule $\mathrm{id}_{X}=\langle X \hookleftarrow X \hookrightarrow X\rangle$.

The semantics of a program $P$ with interface $X$, denoted by $\llbracket P \rrbracket$, is a set of triples such that, for all $\langle g, h, i\rangle \in \llbracket P \rrbracket$, the domain of $g$ and $i$ is $X$ and the codomain of $h$ and $i$ is equal:

(1) $\llbracket \rho \rrbracket \quad$ as in Definition 5 ,

(2) $\llbracket\{P, Q\} \rrbracket=\llbracket P \rrbracket \cup \llbracket Q \rrbracket$,

(3) $\llbracket\langle P ; Q\rangle \rrbracket=\left\{\left\langle g_{1}, h_{2}, i_{2} \circ i_{1}\right\rangle \mid\left\langle g_{1}, h_{1}, i_{1}\right\rangle \in \llbracket P \rrbracket,\left\langle g_{2}, h_{2}, i_{2}\right\rangle \in \llbracket Q \rrbracket, h_{1}=g_{2}\right\}$,

(4) $\llbracket \operatorname{try} P \rrbracket=\{\langle g, h, i\rangle \mid\langle g, h, i\rangle \in \llbracket P \rrbracket\} \cup\{\langle g, g, \mathrm{id}\rangle \mid \nexists h .\langle g, h, i\rangle \in \llbracket P \rrbracket\}$,

$\llbracket P \downarrow \rrbracket=\left\{\langle g, h, \mathrm{id}\rangle \in P^{*} \mid \nexists h^{\prime} .\left\langle h, h^{\prime}, \mathrm{id}\right\rangle \in \llbracket \operatorname{Fix}(P) \rrbracket\right\}$,

where $P^{*}=\bigcup_{j=0}^{\infty} P^{j}$ with $P^{0}=\operatorname{Skip}, P^{j}=\left\langle\operatorname{Fix}(P) ; P^{j-1}\right\rangle$ for $j>0$ and $\llbracket \operatorname{Fix}(P) \rrbracket=\{\langle g, h \circ i, \mathrm{id}\rangle \mid$ $\langle g, h, i\rangle \in \llbracket P \rrbracket\}$. Instead of $\langle g, h, i\rangle \in \llbracket P \rrbracket$, we write $g \Rightarrow_{P, i} h$ or short $g \Rightarrow_{P} h$.

In the following, we consider the basic transformations [6]. The construction Shift "shifts" existential conditions over morphisms into a disjunction of existential application conditions. This can be done because the category of typed graphs has an $\mathscr{E}^{\prime}-\mathscr{M}$-factorization. The construction Left "shifts" a right application condition over a rule into a left application condition. Constraints can be integrated into left application conditions of a rule such that every transformation is condition-preserving.

Lemma 1 (Shift,Left,Pres). In an $\mathscr{M}$-adhesive category $\mathscr{C}$ with $\mathscr{E}^{\prime}-\mathscr{M}$ pair factorization, there are constructions Shift, Left, and Pres such that the following holds. For each condition $d$ over $P$ and every morphism $b: P \hookrightarrow R, n: R \hookrightarrow H, n \circ b \models d \Longleftrightarrow n \models \operatorname{Shift}(b, d)$. For each rule $p=\langle L \hookleftarrow K \hookrightarrow R\rangle$ and each condition ac over $R$, for each $G \Rightarrow_{p, g, h} H, g \models \operatorname{Left}(p$, ac $) \Longleftrightarrow h \models$ ac. For each rule $\rho$ and each condition $d$, a condition ac $=\operatorname{Pres}(\rho, d)$ can be constructed such that the rule $\langle p$, ac $\rangle$ is $d$-preserving, i.e., for all $g \Rightarrow\langle\rho$, ac $\rangle, g \models d$ implies $h \models d$.

A pair $\left(a^{\prime}, b^{\prime}\right)$ of morphisms is jointly surjective if for each $x \in R^{\prime}$ there is a preimage $y \in R$ with $a^{\prime}(y)=x$ or $z \in C$ with $b^{\prime}(z)=x$. For a rule $p=\langle L \hookleftarrow K \hookrightarrow R\rangle, p^{-1}=\langle R \hookleftarrow K \hookrightarrow L\rangle$ denotes the inverse rule. For $L^{\prime} \Rightarrow_{p} R^{\prime}$ with intermediate graph $K^{\prime},\left\langle L^{\prime} \hookleftarrow K^{\prime} \hookrightarrow R^{\prime}\right\rangle$ is the derived rule.

Construction 1. The construction is as follows.

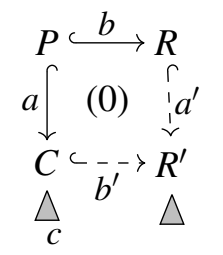

$\operatorname{Shift}(b$, true $):=$ true.

$\operatorname{Shift}(b, \exists(a, c)):=\bigvee_{\left(a^{\prime}, b^{\prime}\right) \in \mathscr{F}} \exists\left(a^{\prime}, \operatorname{Shift}\left(b^{\prime}, c\right)\right)$ where

$\mathscr{F}=\left\{\left(a^{\prime}, b^{\prime}\right) \mid b^{\prime} \circ a=a^{\prime} \circ b, a^{\prime}, b^{\prime}\right.$ inj, $\left(a^{\prime}, b^{\prime}\right)$ jointly surjective $\}$

$\operatorname{Shift}(b, \neg d):=\neg \operatorname{Shift}(b, d), \operatorname{Shift}\left(b, \wedge_{i \in I} d_{i}\right):=\wedge_{i \in I} \operatorname{Shift}\left(b, d_{i}\right)$. 


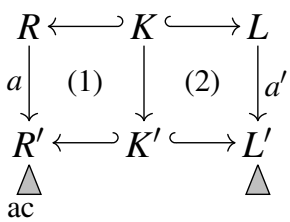

$\operatorname{Left}(p$, true $):=$ true.

$\operatorname{Left}(p, \exists(a, \mathrm{ac})):=\exists\left(a^{\prime}, \operatorname{Left}\left(p^{\prime}\right.\right.$, ac $\left.)\right)$ if $p^{-1}$ is applicable w.r.t. the morphism $a, p^{\prime}:=\left\langle L^{\prime} \hookleftarrow K^{\prime} \hookrightarrow R^{\prime}\right\rangle$ is the derived rule, and false, otherwise. $\operatorname{Left}(p, \neg \mathrm{ac}):=\neg \operatorname{Left}(p, \mathrm{ac}) . \operatorname{Left}\left(p, \wedge_{i \in I} \mathrm{ac}_{i}\right):=\wedge_{i \in I} \operatorname{Left}\left(p, \mathrm{ac}_{i}\right)$.

$\operatorname{Pres}(\rho, d):=\operatorname{Shift}(A \hookrightarrow L, d) \Rightarrow \operatorname{Left}(\rho, \operatorname{Shift}(A \hookrightarrow R, d)$.

Example 2. Let $\rho=\left\langle\mathrm{Pl} \mathrm{Tk} \Rightarrow \mathrm{Pl} 0^{\text {tok }}, \mathrm{Tk}\right\rangle$ be a rule, $d=\nexists\left(\mathrm{Pl} \cdot{ }^{\text {tok }}, \mathrm{Tk}^{\text {tok }} \cdot \mathrm{Pl}\right)$ be a constraint, and $b_{L}$ and $b_{R}$ be the morphisms from the empty graph to the left- and right-hand side of the rule, respectively. Then we have the following.

$$
\begin{aligned}
& \operatorname{Shift}\left(b_{L}, d\right) \quad=\nexists(\underset{1}{\mathrm{Pl}} \cdot *^{\mathrm{tok}} \cdot \underbrace{\mathrm{Tk}}_{2}{ }^{\text {tok }} \cdot \mathrm{Pl}) \wedge \ldots \\
& \operatorname{Shift}\left(b_{R}, d\right) \quad=\nexists(\underset{1}{\mathrm{Pl}} \cdot{ }^{\mathrm{tok}} \cdot \underbrace{\mathrm{Tk}}_{2}{ }^{\text {tok }} \cdot \mathrm{Pl}) \wedge \ldots \\
& \operatorname{Left}\left(\rho, \operatorname{Shift}\left(b_{R}, d\right)\right)=\nexists\left(\mathrm{Pl}_{1}^{\mathrm{Pl}} \quad \mathrm{Tk}_{2}^{\mathrm{tok}^{\mathrm{t}}} \cdot \mathrm{Pl}\right) \wedge \ldots
\end{aligned}
$$

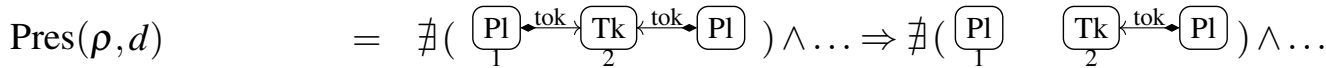

If the rule $\rho$ is equipped with the application condition $\operatorname{Pres}(\rho, d)$, we obtain the rule $\rho^{\prime}=\langle\rho, \operatorname{Pres}(\rho, d)\rangle$, restricting the applicability of the rule to those matches satisfying the application condition and preserving the constraint $d$. The application condition is satisfied if the rule is applied to an occurrence of a graph with Tk-node hat does not have incoming containment edges from different Pl-nodes. (A node of type Tk is said to be Tk-node.)

\section{Repair programs}

In this section, we introduce repair programs and show some repair results for repair programs.

A repair program for a constraint is a graph program with the property that there exists a derivation and the application to any graph yields a graph satisfying the constraint. More generally, we consider repair programs for conditions.

Definition 7 (repair programs). A (typed) program $P$ is a (typed) repair program for a constraint $d$ if, for all (typed) graphs $G, \exists G \Rightarrow_{P} H$ and $\forall G \Rightarrow_{P} H, H \models d$. A program $P$ with interface $A$ is a repair program for a condition ac over $A$, if, for all injective morphisms $g: A \hookrightarrow G, \exists g \Rightarrow_{P, i} h$ and $\forall g \Rightarrow_{P, i} h$, $h \circ i \models$ ac.

Example 3. For the constraint $d$ (see below), intuitively meaning, there do not exist two parallel edges of type tok between a Pl-node and a Tk-node, the program $P_{d}$ is a repair program for $d$.

$$
d=\nexists(\underbrace{\text { tok }}_{\text {tok }}(\mathrm{Tk}) \quad P_{d}=\langle\underbrace{\mathrm{Pl}}_{\text {tok }} \underbrace{\text { tok }}_{\mathrm{Tk}} \Rightarrow \mathrm{Pl} \text { tok } \mathrm{Tk}^{\mathrm{Tk}}\rangle
$$

It works as follows: whenever there are two parallel tok-edges, $P_{d}$ deletes one of the two tok-edges as long as possible. 
We look for stable, maximally preserving, and terminating repair programs. A repair program is stable, if it does nothing whenever the condition is already satisfied, maximally preserving, if, informally, items are preserved whenever possible (see [21]), terminating if the relation $\Rightarrow$ is terminating.

We start with basic conditions of the form $\exists a$ (or $\nexists a$ ) with morphism $a: A \hookrightarrow C$. For basic conditions, we construct so-called repairing sets from the morphism of the condition and repair programs based on the repairing set using the try -statement and the as-long-as-possible iteration, respectively.

Lemma 2 (basic repair). For basic conditions, there are repair programs.

Construction 2. For a real morphism $a: A \hookrightarrow C$, the programs $P_{\exists a}$ and $P_{\nexists a}$ are constructed as follows.

$$
\begin{array}{ll}
P_{\exists a}=\operatorname{try} \mathscr{R}_{a} & \text { with } \mathscr{R}_{a}=\left\{\left\langle b, B \Rightarrow C, \text { ac } \wedge \mathrm{ac}_{B}, a\right\rangle \mid A \hookrightarrow^{b} B \subset C\right\} \\
P_{\nexists a}=\mathscr{S}_{a \downarrow}^{\prime} \downarrow & \text { with } \mathscr{S}_{a}=\left\{\langle a, C \Rightarrow B, b\rangle \mid A \hookrightarrow^{b} B \subset C \text { and }\left(^{*}\right)\right\}
\end{array}
$$

where ac $=\operatorname{Shift}(A \hookrightarrow B, \nexists a), a_{B}=\bigwedge_{B \subset B^{\prime} \subseteq C} \nexists B^{\prime}$, (*) $^{*}$ if $\mathrm{E}_{C} \supset \mathrm{E}_{A}$ then $\left|\mathrm{V}_{C}\right|=\left|\mathrm{V}_{B}\right|,\left|\mathrm{E}_{C}\right|=\left|\mathrm{E}_{B}\right|+$ 1 else $\left|\mathrm{V}_{C}\right|=\left|\mathrm{V}_{B}\right|+1$, and ' denotes the dangling edges operator.

The rules in $\mathscr{R}_{a}$ are of the form $B \Rightarrow C$ where $A \subseteq B \subset C$. They possess an application condition ac requiring the condition $\nexists a$, shifted from $A$ to $B$, and the application condition ac c $_{B}$ requiring that no larger subgraph $B^{\prime}$ of $C$ occurs. The rules in $\mathscr{S}_{a}$ are of the form $C \Rightarrow B$ where $A \subseteq B \subset C$ such that, if the number of edges in $C$ is larger than the one in $A$, they delete one edge and no node, and delete a node, otherwise. By $B \subset C$, both rule sets do not contain identical rules.

Example 4. Consider the condition $d=\exists b$ with $b$ : $\mathrm{Pl} \hookrightarrow \mathrm{Pl} \cdot \mathrm{Tk}$, intuitively meaning that, whenever there is a place there exists a token and a connecting containment edge. Application of the Construction 2 yields a rule set $\mathscr{R}_{b}$ with two rules.

$$
\mathscr{R}_{b}=\left\{\begin{array}{l}
\rho_{1}=\left\langle x_{1}, \mathrm{Pl} \quad \Rightarrow \mathrm{Pl} \cdot{ }^{\mathrm{tok}}, \nexists \mathrm{Tk}, \nexists \mathrm{Pl}, y_{1}\right\rangle \\
\rho_{2}=\left\langle x_{2}, \mathrm{Pl} \mathrm{Tk} \Rightarrow \mathrm{Pl} \cdot{ }^{\mathrm{tok}}, \nexists \mathrm{Tk}, \cdot \mathrm{Pl}, \mathrm{Tk} \wedge \nexists \mathrm{Tk} \mathrm{Pl} \cdot \mathrm{tok}, \mathrm{Tk}, y_{2}\right\rangle
\end{array}\right.
$$

where $x_{1}: \mathrm{Pl} \hookrightarrow \mathrm{Pl}, y_{1}: \mathrm{Pl} \hookrightarrow \mathrm{Pl} \cdot \mathrm{tok}_{\mathrm{Tk}}, x_{2}: \mathrm{Pl} \hookrightarrow \mathrm{Pl} \mathrm{Tk}$, and $y_{2}: \mathrm{Pl} \hookrightarrow \mathrm{Pl} \cdot \mathrm{tok}, \mathrm{Tk}$. The rule $\rho_{1}$ requires a node of type $\mathrm{Pl}$ and attaches a node of type Tk and a connecting containment edge, provided that there do not exist a Pl-node and a Tk-node. The second rule $\rho_{2}$ requires an occurrence of a Pl- and a Tk-node and inserts a connecting containment edge, provided there is no containment edge from the occurrence of the Pl-node to the image of Tk-node, and there is no containment edge to another Tk-node. By Lemma2 2 resp. Theorem 1(2), $P_{d}=\operatorname{try} \mathscr{R}_{b}$ is a repair program for $d=\exists b$.

Conditions with alternating quantifiers ending with true or of the form $\exists(a, \nexists b)$ or $\nexists b$ are proper. A proper condition of the form $\forall(a, c)$ and $\exists(a, c)$ that ends with true is universal and existential, respectively. A condition of the form $\exists a$ ( $\nexists a$ ) is positive (negative).

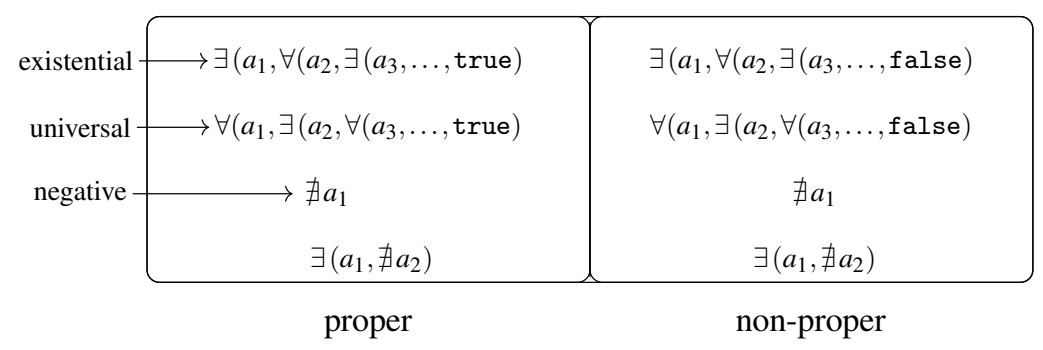


For proper conditions, a repair program can be constructed.

Theorem 1 (Repair I). There is a repair program for proper conditions.

Construction 3. For proper conditions $d$, the repair program $P_{d}$ is constructed inductively as follows.

(1) For $d=$ true, $P_{d}=$ Skip.

(2) For $d=\exists a, P_{d}=\operatorname{try} \mathscr{R}_{a}$.

(3) For $d=\nexists a, P_{d}=\mathscr{S}_{a}^{\prime} \downarrow$.

(4) For $d=\exists(a, c), P_{d}=P_{\exists a} ;\left\langle\operatorname{Mark}(a) ; P_{c} ; \operatorname{Unmark}(a)\right\rangle$.

(5) For $d=\forall(a, c), P_{d}=\left\langle\operatorname{Mark}(a, \neg c) ; P_{c} ; \operatorname{Unmark}(a)\right\rangle \downarrow$.

where $a$ : $A \hookrightarrow C$ is real, $\mathscr{R}_{a}$ and $\mathscr{S}_{a}^{\prime}$ are the sets according to Construction 2 , and $P_{c}$ is a repair program for $c$ with interfaces $C$. $\operatorname{Mark}(a)=\left\langle a, \operatorname{id}_{C}\right\rangle$ is the rule with left interface $a$ and identical plain rule id $\mathrm{id}_{C}=$ $\langle C \hookleftarrow C \hookrightarrow C\rangle$. Given an occurrence of $A$, it is used for a marking of an occurrence of $C$, extending the occurrence of $A$. Similar, $\operatorname{Mark}(a, \operatorname{ac})=\left\langle a, \operatorname{id}_{C}\right.$, ac $\rangle$ is used for marking an occurrence of $C$ satisfying the condition ac. Unmark $(a)=\left\langle\operatorname{id}_{C}, a\right\rangle$ is the identical plain rule with right interface $a$, used for unmarking the occurrence of $C$.

Example 5. Given the constraint $d=\forall(\mathrm{Pl}, \exists \mathrm{Pl}, \mathrm{tok}, \mathrm{Tk})$, meaning that, for each place, there exists a token, a repair program for $d$ can be constructed according to Theorem 11. The constraint $d$ is of the form $\forall(a, c)$ with morphism $a: \emptyset \hookrightarrow \mathrm{Pl}$ and condition $c=\exists \mathrm{Pl} \hookrightarrow \mathrm{Pl}$. tok $\mathrm{Tk}$. By Theorem 1(5), $P_{d}=\left\langle\operatorname{Mark}(a, \neg c) ; P_{c}\right.$; Unmark $\left.(a)\right\rangle \downarrow$. The condition $c$ is of the form $\exists b$. By Repair Theorem 1(2), the repair program for $c$ is $P_{c}=\operatorname{try} \mathscr{R}_{b}$, where $\mathscr{R}_{b}$ is the rule set from Example 4 . The program $P_{d}$ marks an occurrence of a Pl-node without connecting containment edge to a Tk-node. The program $P_{c}$ tries to add a Tk-node and the containment edge, provided there do not exist a Pl-node and a Tk-node, and to add a containment edge between a Pl- and Tk-node, provided that there does not exist such an edge to another Tk-node. Finally the marked part is unmarked. This is done as long as possible. Whenever no further application is possible, the constraint $d$ is satisfied.

The constructed programs are increasing (decreasing): A program $P$ is decreasing (increasing) if all rules in $P$ are decreasing (increasing). A rule $\rho=\langle L \hookleftarrow K \hookrightarrow R$, ac $\rangle$ is decreasing if $L \supset K \cong R$ and increasing if $L \cong K \subset R$.

Fact 1. For negative conditions, the repair program is decreasing. For positive, existential, and universal conditions, the repair program is increasing.

Proof (of Theorem 1). In [7, Theorem 1] the statement is proven for graphs. The statement also holds for typed graphs: For every morphism $a: A \hookrightarrow C$, and every proper subgraph $B$ of $C$, define type $e_{B}=$ type $_{C} \circ$ inc $_{B}$, where for $B \subseteq C$, inc $B$ (short $i_{B}$ ) denotes the inclusion of $B$ in $C$. Then $t_{y p e_{A}}=$ type $_{B} \circ$ inc $_{A}$ and the rules $B \Rightarrow C \in \mathscr{R}_{a}$ and $C \Rightarrow B \in \mathscr{S}_{a}$ consist of typed graph morphisms. In this way the graphs in the rules in $\mathscr{R}_{a}$ and $\mathscr{S}_{a}$ become typed. The typing of the conditions is a direct consequence.

Remark. Theorem 1 could be formulated for a larger class of conditions. In Construction 3 , it is not necessary that the condition $c$ in a condition $\exists(a, c)$ (or $\forall(a, c)$ ) is proper. The construction of a repair program can be done provided that there exists a repair program for $c$. Properness only guarantees the existence of a repair program. 
In the following, we consider conjunctions of conditions. We try to construct a repair program for a conjunction from the repair programs of the conditions in the conjunction.

Given a conjunction $d$ of conditions, we proceed as follows.

(1) Try to find a "preserving sequentialization" $d_{1}, \ldots, d_{n}$ of $d$.

(2) Construct repair programs $P_{1}, \ldots, P_{n}$ for $d_{1}, \ldots, d_{n}$.

(3) Compose the repair programs to a repair program $P=\left\langle P_{1} ; \ldots ; P_{n}\right\rangle$ for $d$.

1. For a conjunction of negative (positive) conditions, this is very simple. We take any sequentialization of the negative (positive) conditions and consider the sequential composition of the corresponding repair programs. This works because, for negative (positive) conditions, the repair programs are decreasing (increasing), and every sequence of decreasing (increasing) repair programs "preserves" the preceding negative (positive) conditions.

2. For conjunctions of universal conditions, this is not so easy: In general, not every sequentialization is preserving. Consider, e.g., the constraints $d_{1}=\forall(\bullet, \exists \bullet \supset)$ and $d_{2}=\forall(\bullet, \exists \bullet \longrightarrow \bullet)$ with the repair programs $P_{1}$ and $P_{2}$ constructed according to Construction 3 . For the sequentialization $d_{1}, d_{2}$, the program $P_{2}$ does not preserve the constraint $d_{1}$ : For a node with loop satisfying $d_{1}$ the program $P_{2}$ adds a new node and a connecting edge. The new node does not have a loop, i.e., the resulting graph does not satisfy $d_{1}$. For the sequentialization $d_{2}, d_{1}$, the program $P_{1}$ preserves the constraint $d_{2}$.

3. Moreover, sometimes there is no preserving sequentialization. Consider, e.g., $d_{1}=\forall(\bullet, \exists \bullet \longrightarrow \bullet)$ and $d_{2}=\forall(\bullet \longrightarrow \bullet, \exists \bullet \longrightarrow \bullet \bullet)$ with the repair programs $P_{1}$ and $P_{2}$ constructed according to Construction 3 , The condition $d_{1} \wedge d_{2}$ is satisfiable: the graph $\bullet \bullet \bullet \bullet \bullet$ satisfies $d_{1} \wedge d_{2}$. Then $P_{2}$ does not preserve $d_{1}$ and $P_{1}$ does not preserve $d_{2}$ : Application of $P_{2}$ to $\bullet \bullet \models d_{1}$ yields to $\bullet \bullet \bullet \forall d_{1}$ and application of $P_{1}$ to $\bullet \models d_{2}$ yields to $\bullet \bullet \bullet \forall d_{2}$.

For this proceeding, preservation of conditions is essential: Whenever a condition is satisfied, it shall be preserved in the following.

Definition 8 (preservation). A program $P$ is $d$-preserving if every rule in $P$ is $d$-preserving.

Lemma 3 (preservation). For every program $P$ and every $d$, there is a $d$-preserving program $P^{d}$.

Construction 4. $P^{d}$ : replace all rules $\rho$ in $P$ by $\langle\rho, \operatorname{Pres}(\rho, d)\rangle$ (Construction 1).

Proof. By Construction 1, all rules in $P^{d}$ are $d$-preserving, thus, the program is $d$-preserving.

In the following, we consider a sequence of conditions together with their repair programs.

Convention. Let $d s=d_{1}, \ldots, d_{n}, P s=P_{1}, \ldots P_{n}$, and $P_{i}$ be a repair program for $d_{i}$, respectively.

A sequence of programs is preserving if for each natural number $k$, the respective repair program $P_{k}$ preserves all preceding conditions, i.e. $P_{1}$ is a repair program for $d_{1}, P_{2}$ is a repair program for $d_{2}$ and $d_{1}$-preserving, $P_{3}$ is a repair program for $d_{3}$ and $d_{1} \wedge d_{2}$-preserving, and so on.

Definition 9 (preservation). The sequence $P s$ is $d s$-preserving (and the sequence $d s$ is preserving) if, for $k=2, \ldots, n, P_{k}$ is $\wedge_{i=1}^{k-1} d_{i}$-preserving. 
We show that sequences of repair programs for sequences of conditions can be sequentially composed to a repair program for the conjunction, provided that the sequences of conditions is preserving.

Lemma 4 (preserving repair). If $d_{1}, \ldots, d_{n}$-preserving, then $\left\langle P_{1} ; \ldots ; P_{n}\right\rangle$ is a repair program for $\wedge_{i=1}^{n} d_{i}$.

Proof. By induction on the number $n$ of conditions with the repair programs. For $n=1$, by Theorem 1, $P_{1}$ is a repair program for $d_{1}$. Inductive hypothesis: If $P_{2}, \ldots, P_{n}$ is $d_{1}, \ldots, d_{n}$-preserving, then $P=\left\langle P_{1} ; \ldots ; P_{n}\right\rangle$ is a repair program for the conjunction $\wedge_{i=1}^{n} d_{i}$. Inductive step: For $n=n+1$, let $P_{2}, \ldots, P_{n+1}$ be $d_{1}, \ldots, d_{n+1}$-preserving. Then $P_{2}, \ldots, P_{n}$ is $d_{1}, \ldots d_{n}$-preserving and, by induction hypothesis, the program $P=\left\langle P_{1} ; \ldots ; P_{n}\right\rangle$ is a repair program for the conjunction $d=\wedge_{i=1}^{n} d_{i}$. Moreover, $P_{n+1}$ is a $d$-preserving repair program for $d_{n+1}$. Consequently, for every transformation $g \Rightarrow_{P} g_{n} \Rightarrow_{P_{n+1}} h, g_{n} \models d$ and $h \models \wedge_{i=1}^{n+1} d_{i}$. Thus, $\left\langle P_{1} ; \ldots ; P_{n+1}\right\rangle$ is a repair program for $\wedge_{i=1}^{n+1} d_{i}$.

A sequence $d s$ of conditions is negative (or positive, or universal) if all conditions in it have the property. For a sequence of negative (or positive) conditions, the sequence $P s$ of repair programs is $d s$-preserving.

Fact 2. If $d s$ is negative (or positive), then $P s$ is $d s$-preserving.

In the following, we consider a conjunction of negative and universal conditions. Let $e_{1}$ be the conjunction of negative and $e_{2}$ the conjunction of universal conditions. By Fact 2 , every sequence $d s_{1}$ of negative conditions is preserving and, by Lemma 4 , the sequential composition $Q_{1}=\left\langle P_{1} ; \ldots ; P_{k}\right\rangle$ of the repair programs forms a repair program for $e_{1}$. In general, not every sequentialization $d s_{2}$ of universal conditions is preserving. We have to require preservation. In the case of preservation, the sequential composition $Q_{2}=\left\langle P_{k+1} ; \ldots ; P_{n}\right\rangle$ of the repair programs forms a repair program for $e_{2}$. But the repair program $Q_{2}$ may be not $e_{1}$-preserving. By Lemma 5 below, $Q_{2}$ can be modified to an $e_{1}$-preserving repair program $Q_{2}^{\prime e_{1}}$ for $e_{2}$. The idea is to delete all occurrences of the morphism $a$ of the universal condition $\forall(a, c)$, which violate the condition $c$. Given a universal condition, we mark an occurrence of the morphism violating the condition, then the occurrence of the morphism at that position is deleted. To mark the morphism at that position, we modify the left interface to the identity of the codomain of the morphism. For a conjunction of universal conditions, an $e_{1}$-preserving repair program can be constructed from the $e_{1}$-preserving program by destroying all non-repaired occurrences of all the universal conditions. By Lemma 4, the program $\left\langle Q_{1}, \mathrm{Q}_{2}^{\prime e_{1}}\right\rangle$ becomes a repair program for $e_{1} \wedge e_{2}$.

A conjunction is negative (universal) if all conditions in the conjunction are negative (universal).

Lemma 5 (preserving repair program). If $Q_{2}$ is a repair program for a preserving universal conjunction $e_{2}$ and $e_{1}$ is a negative conjunction, then there is an $e_{1}$-preserving repair program $Q_{2}^{\prime e_{1}}$ for $e_{2}$.

Construction 5. For a repair program $P$ for $\forall(a, c)$, let $P^{\prime e_{1}}=\left\langle P^{e_{1}} ; P_{\nexists a}^{\text {id }}\right\rangle$ where $P_{\nexists a}^{\text {id }}=\left\langle\operatorname{Mark}(a, \neg c) ; \mathscr{S}_{a}^{\text {id }}\right\rangle \downarrow$ and $\mathscr{S}_{a}^{\text {id }}$ is obtained from $\mathscr{S}_{a}$ by replacing the left interface morphism $a: A \hookrightarrow C$ by the identity id: $C \hookrightarrow C$. For a preserving conjunction $e_{2}$ of universal conditions with sequentialization $d_{1}, \ldots, d_{n}$ and repair program $Q_{2}=\left\langle P_{1} ; \ldots ; P_{n}\right\rangle$, let $Q_{2}^{\prime e_{1}}=\left\langle P_{1}^{\prime e_{1}} ; \ldots ; P_{n}^{\prime e_{1}}\right\rangle$.

Proof. 1. For a universal condition $\forall(a, c)$ with $a: A \hookrightarrow C$, the programs $P$ and $P^{e_{1}}$ are increasing. By the $e_{1}$-preserving application condition, whenever the increasing program $P^{e_{1}}$ is not a repair program, the condition $c$ is not satisfied, and the decreasing program $P_{\nexists a}^{\text {id }}$ becomes applicable and destroys all occurrences that do not satisfy the condition $c$. Since $e_{1}$ is a conjunction of negative conditions, $P_{\nexists a}^{\text {id }}$ is $d_{1}$-preserving. Consequently, $P^{\prime e_{1}}$ is $e_{1}$-preserving. Then $P^{\prime e_{1}}$ is a repair program for $d$ : For every occurrence of $a$, the occurrence is either (1) repaired by $P^{e_{1}}$ or (2) destroyed by $P_{\nexists a}^{\text {id }}$. 
2. By assumption, $d_{1}, \ldots, d_{n}$ is preserving. Moreover, for $i=1, \ldots, n, P_{i}$ is repair program for $d_{i}$ and, by Lemma $51, P_{i}^{\prime e_{1}}$ is an $e_{1}$-preserving repair program for $d_{i}$. Since $d_{1}, \ldots, d_{n}$ is preserving and by Lemma $4, Q_{2}^{\prime e_{1}}=\left\langle P_{1}^{\prime e_{1}} ; \ldots ; P_{n}^{\prime e_{1}}\right\rangle$ is a repair program for $\wedge_{i=1}^{n} d_{i}=e_{2}$. Since all programs in the sequential composition are $e_{1}$-preserving, the program $Q_{2}^{\prime e_{1}}$ is $e_{1}$-preserving. Consequently, every transformation $g \Rightarrow_{Q} m$ is of the form $g \Rightarrow_{Q_{1}} h \Rightarrow_{Q_{2}^{\prime e_{2}}} m$. Since $Q_{1}$ is a repair program for $e_{1}, h \models e_{1}$. Since $Q_{2}^{\prime e_{2}}$ is the $e_{1}$-preserving repair program for $e_{2}, m \models e_{1} \wedge e_{2}$. Thus, $Q$ is a repair program for $e_{1} \wedge e_{2}$.

Fact 3 (composition). For arbitrary conditions $e_{1}, e_{2}$, the following holds. If $Q_{1}$ is a repair program for $e_{1}$ and $Q_{2}^{\prime e_{1}}$ be an $e_{1}$-preserving repair program for $e_{2}$, then $\left\langle Q_{1}, Q_{2}^{\prime e_{1}}\right\rangle$ a repair program for $e_{1} \wedge e_{2}$.

A sequence $d s=d_{1}, \ldots, d_{n}$ is negative (or positive, or existential, or universal) if all $d_{i}$ are negative (or positive, or existential, or universal). In the following, $d s_{1}=d_{1}, \ldots, d_{k}, d s_{2}=d_{k+1}, \ldots, d_{n}$ with conjunction $e_{1}=\wedge_{i=1}^{k} d_{i}$ and $e_{2}=\wedge_{i=k+1}^{n} d_{i}$.

The following theorem says under which conditions a repair program for a conjunction of conditions can be constructed from the repair programs of its components.

Theorem 2 (Repair II). There is a repair program $P$ for a conjunction $d=\wedge_{i=1}^{n} d_{i}$ of conditions provided that $d$ is satisfiable, there are repair programs $P_{1}, \ldots, P_{n}$ for $d_{1}, \ldots, d_{n}$, respectively, and there is a sequentialization $d s=d_{1}, \ldots, d_{n}$, and

1. $d s$ is negative, or positive, or preserving,

2. $d s_{1}$ is positive, and $d s_{2}$ is existential (or universal) \& preserving.

3. $d s_{1}$ is negative, and $d s_{2}$ is universal \& preserving.

\section{Construction 6.}

1. For negative (or positive, or preserving) $d s$, let $P=\left\langle P_{1} ; \ldots ; P_{n}\right\rangle$.

2. For positive $d s_{1}$, universal (or existential) \& preserving $d s_{2}$, let $P=\left\langle Q_{1} ; Q_{2}\right\rangle$.

3. For negative $d s_{1}$, universal \& preserving $d s_{2}$, let $P=\left\langle Q_{1} ; Q_{2}^{\prime e_{1}}\right\rangle$.

where $P_{1}, \ldots, P_{n}$ are repair programs for $d_{1}, \ldots, d_{n}$, respectively, $Q_{1}=\left\langle P_{1} ; \ldots ; P_{k}\right\rangle, Q_{2}=\left\langle P_{k+1} ; \ldots ; P_{n}\right\rangle$, and $Q_{2}^{\prime e_{1}}=\left\langle P_{k+1}^{\prime e_{1}} ; \ldots ; P_{n}^{\prime e_{1}}\right\rangle$ where $e_{1}=\wedge_{i=1}^{k} d_{i}$ and $e_{2}=\wedge_{i=k+1}^{n} d_{i}$.

Example 6. Consider the constraints $d_{1}=\nexists\left(\mathrm{Pl} \cdot{ }^{\mathrm{tok}}, \mathrm{Tk} \cdot \mathrm{tok} \cdot \mathrm{Pl}\right)$ and $d_{2}=\forall\left(\mathrm{Pl}, \exists\left(\mathrm{Pl} \cdot \mathrm{tak}^{\mathrm{Tk}}\right)\right)$ (see Example 5). By Theorem 1, there are repair programs

$$
\begin{aligned}
& P_{1}=\left\langle\mathrm{Pl}{ }^{\text {tok }} \cdot \mathrm{Tk}^{\text {tok }} \cdot \mathrm{Pl} \Rightarrow \mathrm{Pl} \mathrm{Tk}^{\text {tok }} \cdot \mathrm{Pl}\right\rangle \downarrow \\
& P_{2}=\left\langle\operatorname{Mark}(a, \neg c) ; \operatorname{try} \mathscr{R}_{b} ; \operatorname{Unmark}(a\rangle \downarrow\right.
\end{aligned}
$$

where $a: \emptyset \hookrightarrow \mathrm{PI}, c=\exists \mathrm{Pl} \hookrightarrow \mathrm{PI} \cdot \mathrm{tok}, \mathrm{Tk}$, and $\mathscr{R}_{b}$ as in Example 4

By Theorem 2, there is a repair program $P=\left\langle P_{1} ; P_{2}^{\prime d_{1}}\right\rangle$ for $d=d_{1} \wedge d_{2}$. By Lemma 3 , the $d_{1}$-preserving version $P_{2}^{d_{1}}$ of $P_{2}$ is obtained from the repair program $P_{2}$ by equipping each rule $\rho$ in $\mathscr{R}_{b}$ with application condition $\operatorname{Pres}\left(\rho, d_{1}\right)$. For $\rho_{2} \in \mathscr{R}_{b}, \rho_{2}^{\prime}=\left\langle\rho_{2}, \operatorname{Pres}\left(\rho_{2}, d_{1}\right)\right\rangle=\nexists\left(\mathrm{Pl} \cdot{ }^{\text {tok }}, \mathrm{Tk}, \mathrm{tok} \cdot \mathrm{Pl} \wedge \ldots\right.$ $\nexists\left(\mathrm{Pl}\right.$ Tk $\left.{ }^{\text {tok }} \cdot \mathrm{Pl}\right) \wedge \ldots$ The program $P_{2}^{d_{1}}$ is not a repair program for $d_{2}: P_{2}^{d_{1}}$ is not applicable to the graph $G$ : $\mathrm{Pl} \quad \mathrm{Tk}$ tok $\mathrm{Pl}$ and $G \not \models d_{2}$.

By Lemma 5, there is a $d_{1}$-preserving repair program $P_{2}^{\prime d_{1}}=\left\langle P_{2}^{d_{1}} ; P_{\nexists a}^{\mathrm{id}}\right\rangle$ for $d_{2}$ where $P_{\nexists a}^{\mathrm{id}}$ is a slightly modified version of the repair program $P_{\nexists a}$ for the condition $\nexists a$. In more detail, the program looks 
as follows: $P_{\nexists a}^{\text {id }}=\left\langle\operatorname{Mark}\left(\mathrm{Pl}, \nexists(\mathrm{Pl}\right.\right.$, tok $\left.\mathrm{Tk}) ;\langle x, \mathrm{Pl} \Rightarrow \emptyset\rangle^{\prime}\right\rangle \downarrow$ where $x$ is the identity $x: \mathrm{Pl} \hookrightarrow \mathrm{Pl}$. The program marks an occurrence of the Pl-node without incoming containment edge from a Tk-node and deletes (in SPO-style) the occurrence of the Pl-node; this is done as long as possible. In this way, Pl-nodes not satisfying the condition $c$, are deleted. We obtain a repair program for $d_{1} \wedge d_{2}$.

Proof. 1. Let $d_{1}, \ldots, d_{n}$ be negative (positive). Then the repair programs $P_{1}, \ldots, P_{n}$ are decreasing (increasing) and $d_{1}, \ldots, d_{n}$ is preserving. Then, by Lemma $4,\left\langle P_{1} ; \ldots ; P_{n}\right\rangle$ is a repair program for $\wedge_{i=1}^{n} d_{i}$. 2. Let $d_{1}, \ldots, d_{k}$ be positive and $d_{k+1}, \ldots, d_{n}$ universal (or existential) and preserving. By Theorem 2,1 there are repair programs $Q_{1}=\left\langle P_{1} ; \ldots ; P_{k}\right\rangle$ and $Q_{2}=\left\langle P_{k+1} ; \ldots ; P_{n}\right\rangle$ for $e_{1}=\wedge_{i=1}^{k} d_{i}$ and $e_{2}=\wedge_{i=k+1}^{n} d_{i}$, respectively. Since $Q_{2}$ is increasing, it is $e_{1}$-preserving. By Lemma $4,\left\langle Q_{1} ; Q_{2}\right\rangle$ is a repair program for $e_{1} \wedge e_{2}=\bigwedge_{i=1}^{n} d_{i}$.

3. Let $d_{1}, \ldots, d_{k}$ be negative and $d_{k+1}, \ldots, d_{n}$ universal and preserving. By Theorem 2.1 there are repair programs $Q_{1}=\left\langle P_{1} ; \ldots ; P_{k}\right\rangle$ and $Q_{2}=\left\langle P_{k+1} ; \ldots ; P_{n}\right\rangle$ for $e_{1}=\wedge_{i=1}^{k} d_{i}$ and $e_{2}=\wedge_{i=k+1}^{n} d_{i}$, respectively. By Lemma 5, $Q_{2}^{\prime e_{1}}$ is the $e_{1}$-preserving repair program for $e_{2}$. By Fact 3 , $\left\langle Q_{1} ; Q_{2}^{\prime e_{1}}\right\rangle$ is a repair program for $e_{1} \wedge e_{2}=\bigwedge_{i=1}^{n} d_{i}$. An illustration of this part of the proof is given in Figure 4 .

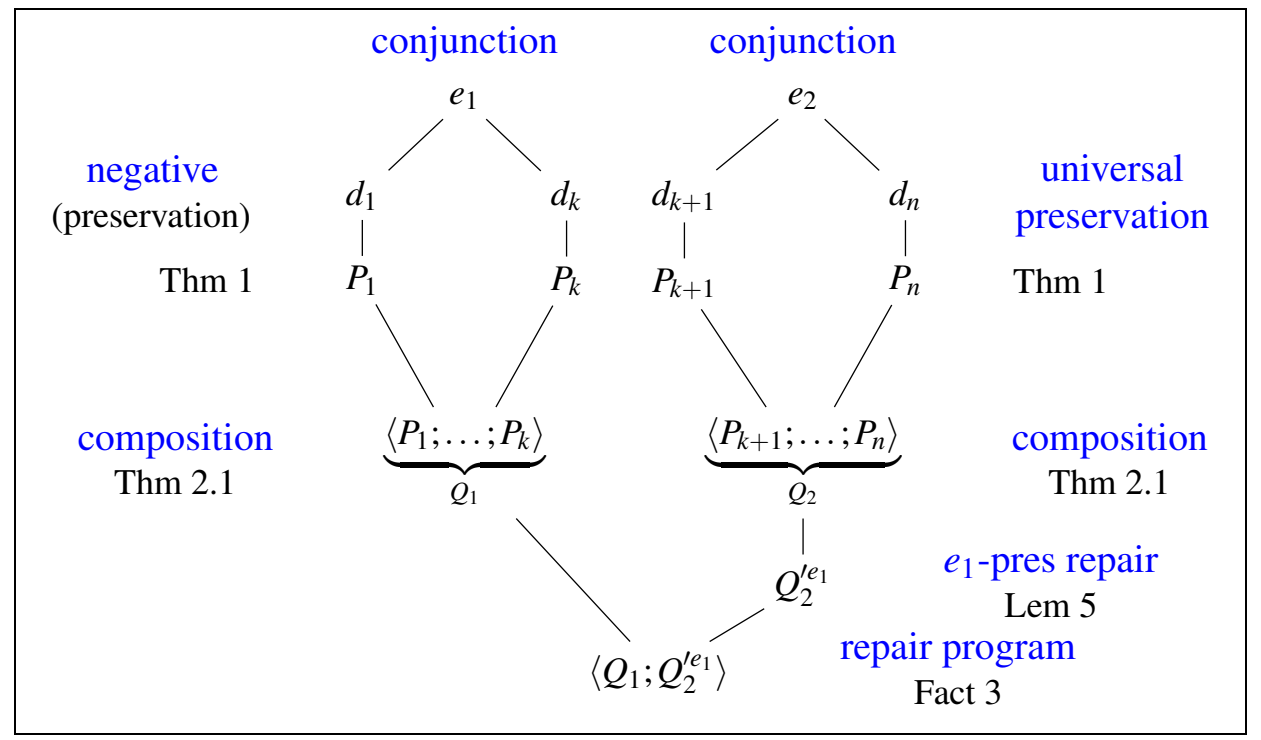

Figure 4: Illustration of the proof of Theorem 23

In the following, we consider disjunctive conditions, i.e. disjunctions of conditions. Whenever there exists a repair program for one of the subconditions, this repair program can be used for the disjunctive conditions as well. Every repair program for a condition is also a repair program for the corresponding disjunctive condition.

\section{Theorem 3 (Repair III).}

1. If $P$ is a repair program for $d$ and $d \Rightarrow d^{\prime}$, then $P$ is a repair program for $d^{\prime}$.

2. Every repair program for $d_{1}$ is repair program for $\bigvee_{i=1}^{n} d_{i}$.

3. If $P_{1}, \ldots, P_{n}$ are repair programs for $d_{1}, \ldots, d_{n}$, then $\left\{P_{1}, \ldots, P_{n}\right\}$ is a repair program for $\bigvee_{i=1}^{n} d_{i}$. 
Proof. 1. If $P$ is a repair program for $d$ and $d \Rightarrow d^{\prime}$, then for every transformation $g \Rightarrow_{P} h, h \models d \Rightarrow d^{\prime}$, i.e., $P$ is a repair program for $d^{\prime}$. 2. By $d_{1} \Rightarrow \bigvee_{i=1}^{n} d_{i}$ and statement $1, P_{1}$ is a repair program for $\bigvee_{i=1}^{n} d_{i}$. 3. Since $d_{i} \Rightarrow \bigvee_{i=1}^{n} d_{i}$ and by statement 1 and $2,\left\{P_{1}, \ldots, P_{n}\right\}$ is a repair program for $\bigvee_{i=1}^{n} d_{i}$

There are repair programs for a large class of conditions: for all proper and generalized-proper ones, preserving conjunctions, and disjunctions. In this context, a condition is said to be generalized proper, if it is obtained form a proper one by replacing a subcondition by a condition (over the same graph) with a repair program.

Definition 10 (generalized proper). Let $d=\mathrm{Q}(a, c)$ be a proper condition. A generalized proper condition $d^{\prime}=\left[c / c^{\prime}\right]$ is obtained by replacing $c$ with a condition $c^{\prime}$, provided there exists a repair program for $c^{\prime}$.

Definition 11 (legit conditions). The class of legit conditions is defined inductively as follows.

(1) If $d$ is proper or generalized proper, then $d$ is legit.

(2) If $d_{1}, \ldots, d_{n}$ are legit and preserving, then $\bigwedge_{i=1}^{n} d_{i}$ is legit.

(3) If $d_{1}$ is legit, then $\bigvee_{i=1}^{n} d_{i}$ is legit.

Theorem 4 (Repair for legit conditions). For legit conditions, there is a repair program.

Proof. By induction of the structure of conditions. Let $d$ be legit.

(1) If $d$ is proper, then, by Theorem 1, there is a repair program for $d$.

If $d$ is generalized proper, then $d$ is of the form $\mathrm{Q}(a, c)$ where $c$ is legit. By induction, there is a repair program for $c$. By a generalized Theorem 1, there is a repair program for $d$. (2) Let $d s=d_{1}, \ldots, d_{n}$ are legit and $d s$ preserving. By induction hypothesis, there are repair programs for $d_{1}, \ldots, d_{n}$. By Theorem 2 , there is a repair program for the conjunction $\bigwedge_{i=1}^{n}$. (3) Let $d_{1}$ be legit. By induction hypothesis, there is a repair program for $d_{1}$. By Theorem 3 , there is a repair program for $\bigvee_{i=1}^{n} d_{i}$. This completes the inductive proof.

As a consequence of Construction 3, we obtain the following.

Lemma 6 (program properties). The repair programs for proper conditions based on Construction 3 are (1) stable, (2) maximally preserving, (3) and terminating.

Proof. Let $d$ be a proper condition and $P_{d}$ the program in Construction 3. (1) By the application condition ac $=\operatorname{Shift}(A \hookrightarrow B, \nexists a)$, a rule in $\mathscr{R}_{a}$ can only be applied, iff the condition is not satisfied. By the semantics of Skip, try, and $\downarrow$, the repair programs are stable. The proof of (2) and (3) can be found in [21] and [7], respectively.

Lemma 7 (program properties). The repair programs for legit conditions as above are stable and terminating.

Proof. By induction on the structure of conditions. Let $d$ be a legit condition and $P_{d}$ be the corresponding repair program.

(1) If $d$ is proper, then by Lemma 6, the repair program $P_{d}$ is stable and terminating. If $d$ is generalized proper, then, by induction hypothesis, $P_{d}$ is stable and terminating. 
(2) By induction hypothesis, $P_{i}, P_{i}^{e_{1}}$ are stable and terminating, $\left\langle P_{1} ; \ldots ; P_{n}\right\rangle$, and $Q_{1}, Q_{2}$ are stable and terminating. Finally $Q_{2}^{\prime e_{1}}=\left\langle P_{k+1}^{\prime e_{1}} ; \ldots ; P_{n}^{\prime e_{1}}\right\rangle$, with $P_{j}^{\prime e_{1}}=\left\langle P_{j}^{e_{1}} ; P_{\nexists a, j}^{\text {id }}\right\rangle$, is (a) stable and (b) terminating: (a) By Construction, $P_{\nexists a, j}^{\text {id }}$ is only applicable, iff the condition is not satisfied. By the semantics of $\downarrow$, it is stable. (b) By Construction, $P_{\nexists a, j}^{\mathrm{id}}$ is decreasing, consequently it is terminating. Consequently, $\left\langle Q_{1} ; Q_{2}^{\prime e_{1}}\right\rangle$ is stable and terminating.

(3) If $d=\bigvee_{i}^{n} d_{i}$, then, by induction hypothesis, $P_{1}$ is stable and terminating. Consequently, $\left\{P_{1}, \ldots, P_{n}\right\}$ is stable and terminating.

Remark (Implementation). The approach to graph repair has been implemented in ENFORCE+.

\section{Application to meta-modeling}

The standard tool for model-driven engineering is the Eclipse Modeling Framework (EMF). In [2], an EMF model graph is defined as a typed graph, representing the model, satisfying the following conditions: No node has more than one container. There are no two parallel edges of the same type. No cycles of containment occur. For all edges in the opposite edges relation, there exists an edge in opposite direction.

Definition 12 (EMF-model graph). A typed graph $G$ is an EMF-model graph, if it satisfies the following conditions:

1. At most one container

2. No containment cycle

3. No parallel edges

4. All opposite edges

$$
\begin{aligned}
& \forall e_{1}, e_{2} \in C_{G} \cdot \mathrm{t}_{G}\left(e_{1}\right)=\mathrm{t}_{G}\left(e_{2}\right) \text { implies } e_{1}=e_{2} \\
& \forall v \in V_{G} \cdot(v, v) \notin \operatorname{cont}_{G} \\
& \forall e_{1}, e_{2} \in E_{G} \cdot \quad \mathrm{s}_{G}\left(e_{1}\right)=\mathrm{s}_{G}\left(e_{2}\right), \mathrm{t}_{G}\left(e_{1}\right)=\mathrm{t}_{G}\left(e_{2}\right), \text { and } \\
& \text { type }_{E_{G}}\left(e_{1}\right)=\text { type }_{E_{G}}\left(e_{2}\right) \text { implies } e_{1}=e_{2} \\
& \forall\left(e_{1}, e_{2}\right) \in O . \quad \forall e_{1}^{\prime} \in E_{G} \cdot t y p e_{G}\left(e_{1}^{\prime}\right)=e_{1} . \quad \exists e_{2}^{\prime} \in E_{G} . \\
& \text { type }_{G}\left(e_{2}^{\prime}\right)=e_{2}, \mathrm{~s}_{G}\left(e_{1}^{\prime}\right)=\mathrm{t}_{G}\left(e_{2}^{\prime}\right) \text { and } \mathrm{s}_{G}\left(e_{2}^{\prime}\right)=\mathrm{t}_{G}\left(e_{1}^{\prime}\right)
\end{aligned}
$$

The set $C_{G}$ denotes the set of edges in $G$ which are typed by a containment edge. $\operatorname{cont}_{G} \subseteq V_{G} \times V_{G}$ is the containment relation induced by the set $C \subseteq E_{T}$ : If $e \in C$ and $v_{1} \leq \mathrm{s}(e), v_{2} \leq \mathrm{t}(e)$, then $\left(v_{1}, v_{2}\right) \in$ cont $_{G}$. If $\left(v_{1}, v_{2}\right),\left(v_{2}, v_{3}\right) \in \operatorname{cont}_{G}$, then $\left(v_{1}, v_{3}\right) \in \operatorname{cont}_{G}$.

The conditions are said to be EMF-constraints.

The second constraint is a monadic second order constraint. Instead of it, we consider the constraint "No containment cycle of length $\leq k$ " for a fixed natural number $k$. The resulting constraints, called $E M F k$ constraints, are first-order constraints and can be expressed by typed graph constraints [6].

Fact 4 (EMF $k$-constraints). For the EMF $k$-constraints, there is a schema of typed graph constraints:

1. At most one container

2. No containment cycle of length $\leq k$

3. No parallel edges

4. All opposite edges
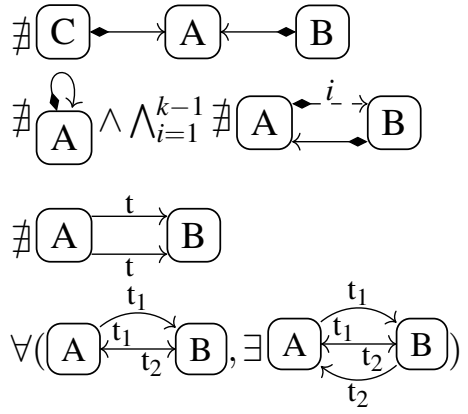
where $\mathrm{A}, \mathrm{B}, \mathrm{C}, \mathrm{t}, \mathrm{t}_{1}, \mathrm{t}_{2}$ are node and edge types, respectively, edges without type are arbitrary typed, and $\rightarrow i_{\rightarrow}$ denotes a path of containment edges of length $i$.

The first constraint requires that there are no two different containment edges with a common target. The second constraint requires that there are no loops and no cycles of length $\leq k$. The third constraint requires, that there are no parallel edges of the same type. The fourth constraint requires that, if there is an opposite-edge marking between an A-typed and a B-typed node with type requirement $t_{1}, t_{2}$, there exists already one edge $e_{1}$ with the type $\mathrm{t}_{1}$, then an opposite edge with type $\mathrm{t}_{2}$ in opposite direction should exist.

Fact 5. The instances of EMF $k$ constraints are negative or universal. Every conjunction of EMF $k$ constraint instances is satisfiable. Every sequence of instances of one EMF $k$ constraint is preserving.

Lemma 8 (preservation). Every conjunction of instances of an EMF $k$ constraint is preserving.

Proof. The instances of the first three EMF $k$ constraints are negative; thus, each conjunction of them is preserving. The instances of the forth EMFk constraint are universal; by induction on the number of constraints, it can be shown that each conjunction is preserving.

Let emf $k_{1}$, emf $k_{2}$ be conjunctions of EMF $k$ constraints. An EMFk model repair program for $\left\langle\mathrm{emf} k_{1}, \mathrm{emf} k_{2}\right\rangle$ is an emf $k_{1}$-preserving repair program for emf $k_{2}$. An EMFk model completion program is an EMF model repair program for true and for the conjunction of all EMF $k$ constraints. A repair program $P$ for a constraint $e$ is stable if, for all transformations $L \Rightarrow_{P} M, L \models e$ implies $L \cong M$, i.e., they do not change the the graph provided the constraint $e$ is satisfied.

Theorem 5 (EMF $k$ model repair \& completion). Let emf $k_{1}$ is a conjunction of negative EMF $k$ constraints, emf $k_{2}$ a conjunction of negative or universal and preserving EMFk constraints, and $\overline{\text { emf } k}$ the conjunction of all EMF $k$ constraints.

1. There is a model-repair program for $\left\langle\mathrm{emf} k_{1}, \mathrm{emf} k_{2}\right\rangle$.

2. There is a model-completion program.

3. The EMF $k$ model repair and completion programs are stable.

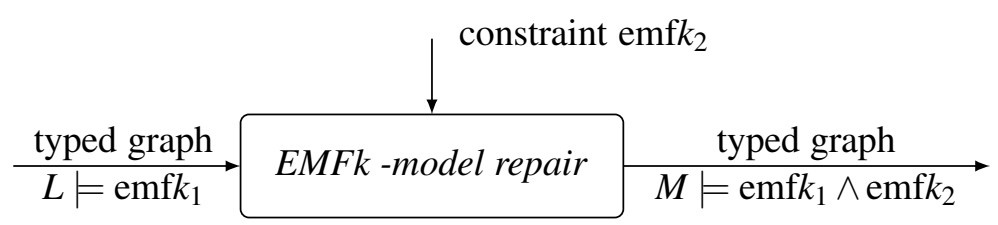

Figure 5: Illustration of EMFk model repair

Proof. 1. By Theorem 11, there are repair program $Q_{1}, Q_{2}$ for emf $k_{1}, \operatorname{emf} k_{2}$, respectively. By Lemma 8 , every conjunction emf $k_{1}$ and emf $k_{2}$ is preserving. Consequently, Lemma 4 can be applied and there is an emf $k_{1}$-preserving repair program $Q_{2}^{\prime \text { emf } k_{1}}$ for emf $k_{2}$, and by Fact $3,\left\langle Q_{1}, Q_{2}^{\prime \text { emf } k_{1}}\right\rangle$ is a repair program for emf $k_{1} \wedge$ emf $k_{2}$.

2. The statement is an immediate consequence of Theorem 5.1 .

3. The statement follows immediately from Construction 3 . 
Remark ((OCL-)Constraints). By the repair results on typed graphs, (model) repair and completion can be done for other constraints satisfying the requirements in Theorem 2 , e.g., for first-order (OCL-)constraints.

Inspecting the EMF $k$ repair (completion) program, it turns out that the program deletes and adds an edge, but it does not change the number of nodes.

Fact 6 (preservation of the number of nodes). The application of the EMF $k$ repair (completion) program does not change the number of nodes.

There is a close relationship between EMF $k$ and EMF. For an EMF $k$ constraint emf $k$, emf denotes the more rigorous EMF constraint requiring no containment cycles and, for an EMF-constraint emf, emf $k$ denotes the weaker EMF $k$ constraint emf requiring no containment cycles of length $\leq k$.

Fact 7 (EMF $k$-EMF). For typed graphs $L$ of node size $\leq k, L \models$ emf $k$ iff $L \models$ emf.

As a consequence, we obtain the following statement for EMF model repair \& completion.

Let $\mathrm{emf}_{1}, \mathrm{emf}_{2}$ be conjunctions of EMF constraints. There is EMF model repair for a typed graph $L \models$ $\mathrm{emf}_{1}$ and $\mathrm{emf}_{2}$ if there is a program $P$ such that, for all transformations $L \Rightarrow_{P} M, M=\mathrm{emf}_{1} \wedge \mathrm{emf}_{2}$. There is an $E M F$ model completion for a typed graph $L$ if there is a program $P$ such that, for all transformations $L \Rightarrow_{P} M, M$ is an EMF-model graph. Model repair and completion for a constraint $e$ are stable if, for all typed graphs $L \models e$, all repairs (completions) yield a typed graph isomorphic to $L$.

Theorem 6 (EMF model repair \& completion). Let $\mathrm{emf}_{1}$ is a conjunction of negative EMF-constraints, $\mathrm{emf}_{2}$ a conjunction of negative or universal and preserving EMF-constraints, and $\overline{\mathrm{emf} k}$ the conjunction of all EMF $k$ constraints.

1. There is a EMF model repair for all typed graphs $L \models \mathrm{emf}_{1}$ and emf $k_{2}$.

2. There is a EMF model completion for all typed graphs $L$.

3. The EMF model repair and completion for a constraint $e$ are stable for all typed graphs $L$.

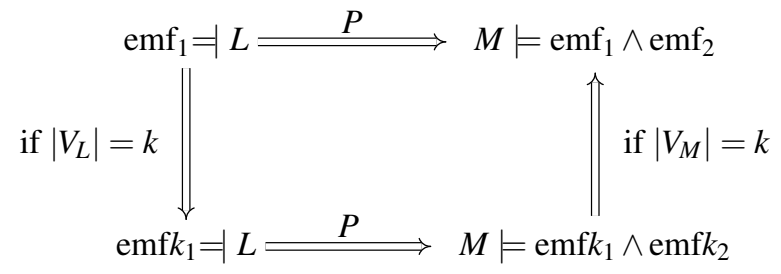

Figure 6: Relation on emf and emfk

Proof. 1. For a typed graph $L$ of node size $k$ satisfying $\mathrm{emf}_{1}$ and $\mathrm{emf}_{2}$, we take the EMF $k$ repair program $P$ for $\left\langle\operatorname{emf} k_{1}, \operatorname{emf} k_{2}\right\rangle$ and apply it to $L$. By Fact $7, L \models \operatorname{emf}_{1}$ implies $L \models \operatorname{emf} k_{1}$. By Theorem 2, the application of $P$ to $L$ yields a typed graph $M$ satisfying emf $k_{1} \wedge$ emf $k_{2}$. The program does not change the number of nodes, i.e., $M$ is a typed graph with $k$ nodes. By Fact $7, M$ satisfies $\mathrm{emf}_{1} \wedge \mathrm{emf}_{2}$.

2. For a typed graph $L$ of node size $k$, we take the EMF $k$-completion program and apply it to $L$ yielding an EMFk -model graph $M$, i.e, a typed graph $M$ satisfying emfk. The program does not change the number of nodes, i.e., $M$ is a typed graph with $k$ nodes. By Fact $7 M$ satisfies $\operatorname{emf}_{1} \wedge \mathrm{emf}_{2}$. 
3. By Theorem 5, the EMFk -model repair and completion programs are stable, i.e., for all transformations $L \Rightarrow_{P} M, L \models e$ implies $L \cong M$. Applying the programs to a typed graph, the property remains preserved.

Remark (Repair of other structures). In this section, the results in Section 3 are applied to metamodeling: typed graphs are repair w.r.t. EMF constraints. Obviously, typed graphs can also repaired w.r.t. other constraints, e.g. OCL-graph constraints as considered in [20]. Note that the presented results hold in every $\mathscr{M}$-adhesive category with $\mathscr{E}^{\prime}-\mathscr{M}$-pair factorization. As a consequence, we can do repair for high-level structures and high-level constraints [4].

Remark (Model generation). In model generation, given a meta-model, one tries to find some (all) instances of the meta-model. Model generation may be seen as a special case of model completion applying the program: For a fixed $k$, the application of the EMF $k$ model completion program to the empty typed graph yields EMF $k$ model graphs. By Fact 6 , every EMF $k$ model graph with node size $\leq k$ is an EMF model graph. In this way, we obtain some instances of the meta-model.

\section{Related work}

In this section, we present some related concepts on model repair, for which there is a wide variety of different approaches. Recently, there has been a sophisticated survey on different model repair techniques, and a feature-based classfication of these approaches, see [11]. In their sense, our approach is stable (see Theorem 5,3).

In Schneider et al. 2019 [22], a logic-based incremental approach to graph repair is presented, generating a sound and complete (upon termination) overview of least changing repairs. The graph repair algorithm takes a graph and a first-order (FO) graph constraint as inputs and returns a set of graph repairs. Given a condition and a graph, they compute a set of symbolic models, which cover the semantics of a graph condition. Both approaches are proven to be correct, i.e. the repair (programs) yield to a graph satisfying the condition. The delta-based repair algorithm takes the graph update history explicitly into account, i.e. the approach is dynamic. In contrast, our approach is static, i.e., we first construct a repair program, then apply this program to an arbitrary graph. The repair algorithm does not terminate, if the repair updates trigger each other ad infinitum. Here, we have constructed terminating repair programs.

In Biermann et al. 2012 [2], for EMF model transformations, consistent transformations are defined. For a set of rules, they slightly modify them, to get so-called consistent transformation rules. This way, a direct transformation step applied at an EMF model yields an EMF model graph again. In our approach, a direct transformation step leads to typed graphs. We use the repair program to complete the typed graph to an EMF model graph.

In Nassar et al. 2017 [13], a rule-based approach to support the modeler in automatically trimming and completing EMF models is presented. For that, repair rules are automatically generated from multiplicity constraints imposed by a given meta-model. The rule schemes are carefully designed to preserve the EMF model constraints. One can use the approach in this paper to transform a typed graph to an EMF $k$ model graph, then, one can use the approach of [13], to transform an EMF model graph to an EMF model graph, satisfying additional multiplicity constraints.

In Nassar et al. 2020 [12], a method to simplify constraint-preserving application conditions is presented. Their simplifications of the application conditions are based on three main concepts: (1) If the elements which are deleted (or added) by a rule are type-disjoint with the types of the constraint, i.e. they share 
no types, the application condition simplifies to true, (2) For increasing (or decreasing) rules, and positive (or negative) constraints, the application is true, (3) For negative constraints $\nexists C$ one may omit the cases where $C$ and the elements created by the rule overlap in at least one element. The simplified application conditions are proven to be logically equivalent to the original application condition. The results are proven to be correct for $\mathscr{M}$-adhesive categories, and can be used to simplify the application conditions needed for our construction of condition-preserving application conditions (Lemma 1), as well. Furthermore, the EMF model constraints (Fact 4) are simplified by replacing every subcondition violating the no parallel edge, and at most one container constraints with false.

In Kosiol et al. 2020 [9] two notions of consistency as a graduated property are introduced: consistencysustaining rules do not change the number of violations of a constraint in a graph, and consistencyimproving rule reduce the number of violations in a graph. The definition is based on the so-called consistency index, given by the number of constraint violations in a graph, divided by the number of "relevant occurrences" of the constraints in a graph. A transformation is consistency sustaining, if the consistency index for the input graph is equal or less than the resulting graph, and consistency improving if the number of the violations in the resulting graph is smaller than in the input graph. A rule is consistency sustaining, if all transformations are. A rule is consistency improving, if all applications of the rule are consistency sustaining, there exists a graph with constraint violations, and a transformation, such that the number of the violations in the resulting graph is smaller than in the input graph. A rule is strongly consistency improving if all its applications to a graph with constraint violations is consistency improving. In their setting, the rules derived from our approach are strongly consistency improving,

In Taentzer et al. 2017 [26], a designer can specify a set of so-called change-preserving rules, and a set of edit rules. Each edit rule, which yields to an inconsistency, is then repaired by a set of repair rules. The construction of the repair rules is based on the complement construction. It is shown, that a consistent graph is obtained by the repair program, provided that each repair step is sequentially independent from each following edit step, and each edit step can be repaired. The repaired models are not necessarily as close as possible to the original model.

In Rabbi et al. 2015 [19], a model completion approach for predicates specified in the Diagrammic Predicate Framework (DPF) is introduced. For every predicate in the model, they derive a set of completion rules, by constructing the pullback of the instance, the meta-model and the graph of the condition. These rules, applied as long as possible, yields a model which conforms to the predicate. In our approach, the rules are derived from the constraint and the meta-model. In both approaches, the meta-model remains unchanged.

In Wang 2016 [28], the semantics of the predicates in the DPF is specified as graph constraints, and a model repair approach for these graph constraints is introduced. For constraints of the form $\forall(L, \exists R)$ or $\forall(L, \nexists R)$, repair rules are directly derived from the constraints. The construction is based on the construction of subgraphs of $L$ and $R$. For the constraint $\forall(L, \exists R)$, for each subgraph $B$, they derive rules $\langle B \Rightarrow R, \nexists R\rangle$ and $\langle L \Rightarrow B, \nexists R\rangle$. For the constraint $\forall(L, \nexists R)$, rules of the form $\langle L \Rightarrow B\rangle$ are derived. The performance of the approach has been optimized for practical application scenarios. In this work, we have combined the programs for proper conditions to a repair program for conjunctions of proper conditions. The properties of the repaired conditions remain preserved, whenever possible. If this is not possible, we delete the occurrence. As far as we can see, the approach in [28] does not handle conjunctions.

In Barriga et al 2019 [1], an algorithm for model repair based on EMF is presented, which relies on reinforcement learning. For each error in the model, a so-called Q-table is constructed, storing a weight 
for each error, and repair action. This weight indicates how good a repair action is, depending on the repair action and regarding the users preferences. The approach can repair errors provided by the EMF diagnostician. The results are not proven but evaluated using mutation testing.

\section{Conclusion}

In this paper, we have presented the theory of typed repair programs, applied it to EMFk -and EMF model graph repair.

1. Typed graph repair. We have extended our results on graph repair to typed graphs. There are repair programs for a large class of conditions, called legit conditions. Application of the repair programs to an arbitrary typed graph yields a typed graph satisfying the condition.

2. EMF $k$ model repair. For EMF $k$ constraints, a first-order variant of EMF constraints, we present stable EMF $k$ model repair and completion programs. Application of these programs to any typed graph yields a repaired typed graph and an EMF $k$ model graph, respectively,

3. EMF model repair. These results are applied to the EMF world and yield to EMF model repair and completion results.

Further topics may be the following.

1. Least changng repairs. Our repair programs induce repairs in the sense of Schneider et al. [22]. It would be nice to show that these induced repairs are least changing repairs.

2. Redirection of edges. Our repair programs try to preserve items; if this is not possible, they delete items. In Nassar et al. [13], multiplicity constraints are considered. In this context, they use the idea, to redirect an edge instead of deleting it. How this can be included in our approach?

3. Generalization to attributed type graphs as e.g. in [20, 16].

Acknowledgements. We are grateful to Annegret Habel, Marius Hubatschek, Jens Kosiol, Okan Özkan, Gabriele Taentzer, and the anonymous reviewers for their helpful comments to this paper.

\section{References}

[1] Angela Barriga, Adrian Rutle \& Rogardt Heldal (2019): Personalized and Automatic Model Repairing using Reinforcement Learning. In: 22nd ACM/IEEE International Conference on Model Driven Engineering Languages and Systems Companion, MODELS Companion, IEEE, pp. 175-181, doi:10.1109/MODELS-C.2019.00030

[2] Enrico Biermann, Claudia Ermel \& Gabriele Taentzer (2012): Formal foundation of consistent EMF model transformations by algebraic graph transformation. Software and System Modeling 11(2), pp. 227-250, doi:10.1007/s10270-011-0199-7

[3] Hartmut Ehrig, Karsten Ehrig, Ulrike Prange \& Gabriele Taentzer (2006): Fundamentals of Algebraic Graph Transformation. EATCS Monographs of Theoretical Computer Science, Springer, doi: $10.1007 / 3-540-31188-2$

[4] Hartmut Ehrig, Ulrike Golas, Annegret Habel, Leen Lambers \& Fernando Orejas (2014): $\mathscr{M}$-Adhesive Transformation Systems with Nested Application Conditions. Part 1: Parallelism, Concurrency and Amalgamation. Mathematical Structures in Computer Science 24, doi:10.1017/S0960129512000357. 
[5] Hartmut Ehrig, Ulrike Golas \& Frank Hermann (2010): Categorical Frameworks for Graph Transformation and HLR Systems based on the DPO Approach. Bulletin of the EATCS 112, pp. 111-121. Available at http://eatcs.org/beatcs/index.php/beatcs/article/view/158

[6] Annegret Habel \& Karl-Heinz Pennemann (2009): Correctness of High-Level Transformation Systems Relative to Nested Conditions. Mathematical Structures in Computer Science 19, pp. 245-296, doi $10.1017 /$ S0960129508007202

[7] Annegret Habel \& Christian Sandmann (2018): Graph Repair by Graph Programs. In: Graph Computation Models (GCM 2018), Lecture Notes in Computer Science 11176, pp. 431-446, doi:10.1007/978-3-030-04771-9_31.

[8] Reiko Heckel \& Gabriele Taentzer (2020): Graph Transformation for Software Engineers - With Applications to Model-Based Development and Domain-Specific Language Engineering. Springer, doi:10.1007/978-3-030-43916-3

[9] Jens Kosiol, Daniel Strüber, Gabriele Taentzer \& Steffen Zschaler (2020): Graph Consistency as a Graduated Property - Consistency-Sustaining and -Improving Graph Transformations. In Fabio Gadducci \& Timo Kehrer, editors: Graph Transformation - 13th International Conference, ICGT, Lecture Notes in Computer Science 12150, Springer, pp. 239-256, doi 10.1007/978-3-030-51372-6_14.

[10] Michael Löwe (1993): Algebraic Approach to Single-Pushout Graph Transformation. Theoretical Computer Science 109, pp. 181-224, doi 10.1016/0304-3975(93)90068-5.

[11] Nuno Macedo, Jorge Tiago \& Alcino Cunha (2017): A Feature-Based Classification of Model Repair Approaches. IEEE Trans. Software Eng. 43(7), pp. 615-640, doi:10.1109/TSE.2016.2620145.

[12] Nebras Nassar, Jens Kosiol, Thorsten Arendt \& Gabriele Taentzer (2020): Constructing optimized constraintpreserving application conditions for model transformation rules. J. Log. Algebraic Methods Program. 114, p. 100564, doi $10.1016 /$ j.jlamp.2020.100564

[13] Nebras Nassar, Hendrik Radke \& Thorsten Arendt (2017): Rule-Based Repair of EMF Models: An Automated Interactive Approach. In: Theory and Practice of Model Transformation (ICMT 2017), Lecture Notes in Computer Science 10374, pp. 171-181, doi:10.1007/978-3-319-61473-1_12.

[14] Christian Nentwich, Wolfgang Emmerich \& Anthony Finkelstein (2003): Consistency Management with Repair Actions. In: Software Engineering, IEEE Computer Society, pp. 455-464, doi:10.1109/ICSE.2003.1201223.

[15] Object Management Group (2014): Object Constraint Language, Version 2.4, OCL (February 2014). Available at https://www .omg.org/spec/OCL/2.4/

[16] Fernando Orejas \& Leen Lambers (2010): Symbolic Attributed Graphs for Attributed Graph Transformation. Electronic Communications of the EASST 30, doi $10.14279 /$ tuj.eceasst.30.405.

[17] Karl-Heinz Pennemann (2009): Development of Correct Graph Transformation Systems. Ph.D. thesis, Universität Oldenburg.

[18] Christopher M. Poskitt \& Detlef Plump (2013): Verifying Total Correctness of Graph Programs. Electronic Communications of the EASST 61, doi 10.14279/tuj.eceasst.61.827

[19] Fazle Rabbi, Yngve Lamo, Ingrid Chieh Yu \& Lars Michael Kristensen (2015): A Diagrammatic Approach to Model Completion. In: Proceedings of the 4th Workshop on the Analysis of Model Transformations, CEUR Workshop Proceedings 1500, CEUR-WS.org, pp. 56-65. Available at http://ceur-ws.org/Vol-1500/paper7.pdf.

[20] Hendrik Radke, Thorsten Arendt, Jan Steffen Becker, Annegret Habel \& Grabriele Taentzer (2018): Translating Essential OCL Invariants to Nested Graph Constraints for Generating Instances of Meta-models. Science of Computer Programming 152, pp. 38-62, doi:10.1016/j.scico.2017.08.006

[21] Christian Sandmann \& Annegret Habel (2019): Rule-based Graph Repair. In: Proceedings Tenth International Workshop on Graph Computation Models, GCM@STAF 2019, EPTCS 309, pp. 87-104, doi:10.4204/EPTCS.309.5. 
[22] Sven Schneider, Leen Lambers \& Fernando Orejas (2019): A Logic-Based Incremental Approach to Graph Repair. In: Fundamental Approaches to Software Engineering - (FASE 2019), Lecture Notes in Computer Science 11424, pp. 151-167, doi:10.1007/978-3-030-16722-6_9.

[23] Shane Sendall \& Wojtek Kozaczynski (2003): Model Transformation: The Heart and Soul of Model-Driven Software Development. IEEE Software 20(5), pp. 42-45, doi:10.1109/MS.2003.1231150.

[24] David Steinberg, Frank Budinsky, Ed Merks \& Marcelo Paternostro (2008): Eclipse Modeling Framework (The Eclipse Series). Addison-Wesley Professional.

[25] Gabriele Taentzer (2012): Instance Generation from Type Graphs with Arbitrary Multiplicities. Electronic Communications of the EASST 47, doi 10.14279/tuj.eceasst.47.727.

[26] Gabriele Taentzer, Manuel Ohrndorf, Yngve Lamo \& Adrian Rutle (2017): Change-Preserving Model Repair. In: Fundamental Approaches to Software Engineering (ETAPS 2017), Lecture Notes in Computer Science 10202, pp. 283-299, doi:10.1007/978-3-662-54494-5_16.

[27] Guido Wachsmuth (2007): Metamodel Adaptation and Model Co-adaptation. In Erik Ernst, editor: ECOOP 2007 - Object-Oriented Programming, 21st European Conference, LNCS 4609, Springer, pp. 600-624, doi:10.1007/978-3-540-73589-228.

[28] Xiaoliang Wang (2016): Towards Correct Modelling and Model Transformation in DPF. Ph.D. thesis, University of Bergen. 